\title{
Can transrectal ultrasound-guided core needle biopsy serve as an accurate diagnostic tool for rectal lesions?
}

\author{
Dong Hoon Baek, Gwang Ha Kim \\ Department of Internal Medicine, Pusan National University School of Medicine and Biomedical Research Institute, Pusan National University \\ Hospital, Busan, South Korea \\ Correspondence to: Gwang Ha Kim. 179, Gudeok-ro, Seo-gu, Busan 49241, South Korea. Email: doc0224@pusan.ac.kr. \\ Provenance: This is an invited article commissioned by the Editorial Office of Annals of Translational Medicine. \\ Comment on: Liu M, Lu ZH, Wang QX, et al. Diagnostic value, safety, and histopathologic discrepancy risk factors for endoscopic forceps biopsy and \\ transrectal ultrasound-guided core needle biopsy in rectum lesions. Ann Transl Med 2019;7:607.
}

Submitted Nov 20, 2019. Accepted for publication Nov 28, 2019.

doi: 10.21037/atm.2019.11.144

View this article at: http://dx.doi.org/10.21037/atm.2019.11.144

Colorectal cancer (CRC) is one of the most common cancers and the leading cause of cancer-related deaths worldwide. The widespread use of colonoscopy in recent times has improved the CRC detection rate; approximately $70 \%$ of CRCs involves the colon and $30 \%$ affects the rectum (1). Endoscopic or surgical resection is essential for confirmation of submucosal (SM) invasion following the diagnosis of rectal cancer. Adenocarcinoma is the most common histopathological type of rectal cancer; however, squamous cell carcinoma, mucinous adenocarcinoma, or neuroendocrine tumor may also occur in the rectum. Treatment and prognosis vary significantly based on the histopathological type of the tumor. The rectum shares limited space with other genitourinary organs, blood vessels, and nerves in the pelvic cavity; therefore, ensuring curative resection of rectal cancer without intraoperative injury to surrounding organs is challenging (2). Consequently, accurate diagnosis of the extent of SM invasion is important to select an optimal treatment modality for rectal cancer.

Early CRC is defined as carcinoma in which tumor invasion is limited to the mucosa or submucosa, regardless of lymph node metastasis (LNM) (3). In contrast to early gastric cancer, early CRC confined to the epithelium or the lamina propria mucosae shows virtually no risk of metastasis because lymphatic and blood vessels are absent in the epithelium and the lamina propria mucosae (4). Endoscopic resection serves as curative therapy in cases of benign adenoma and intramucosal carcinoma (Tis) [corresponding to high-grade intraepithelial neoplasia [HGIN] in western countries] (5-7). Endoscopic resection is indicated for lesions with little risk of LNM (T1 cancer with SM invasion depth $<1,000 \mu \mathrm{m}$ ) in patients with tumors that invade the muscularis mucosae and spread to the submucosa. Surgery should be recommended as radical treatment for CRC categorized as T1b cancers (T1 cancer with SM invasion depth $\geq 1,000 \mu \mathrm{m})$ owing to the high risk of LNM (10\%) (8-11). Therefore, it is important for endoscopists to determine the extent of SM invasion before endoscopic treatment to identify patients in whom endoscopic resection alone can achieve complete cure.

In the current issue, Liu et al. (12) first reported the safety and feasibility of transrectal ultrasound (TRUS)guided core needle biopsy (CNB) for rectal lesions. The authors retrospectively investigated 102 patients with rectal lesions who underwent endoscopic forceps biopsy (EFB) and TRUS-guided CNB prior to surgery. Compared with postoperative pathology findings, the histopathological diagnostic discrepancy rates of EFB and TRUS-guided CNB were $51.0 \%$ (52/102 lesions) and 8.8\% (9/102 lesions), respectively; the kappa value for consistency with postoperative pathology findings was 0.420 for EFB and 0.876 for TRUS-guided CNB. These data suggest that consistency with postoperative pathology findings was better with TRUS-guided CNB than with EFB. Notably, TRUS-guided CNB was more useful to diagnose relatively rare types of malignancies (e.g., squamous cell carcinoma or mucinous adenocarcinoma), as well as benign lesions (e.g., endometriosis). Additionally, the authors described 
characteristic contributors to histopathological discrepancies between EFB and postoperative pathology findings; multivariate analysis showed that flat/depressed lesions and those with thickness $\geq 13.5 \mathrm{~mm}$ were significantly associated with histopathological discrepancies in EFB.

$\mathrm{EFB}$ is a standard technique to diagnose rectal cancers; however, it may not be suitable for optimal evaluation of SM invasion because it has been observed that invasive cancers can be confirmed only in $58.7-67.3 \%$ of rectal tissue samples obtained by EFB prior to surgery (13). Rebiopsy may increase the detection rate of SM invasion; however, this approach may lead to diagnostic delays. Furthermore, even in patients with HGIN diagnosed by $\mathrm{EFB}$, postoperative pathology findings may reveal cancers with invasion of the SM or deeper layers (14). In that TRUS-guided CNB can harvest the five-layer structure of the rectal wall, Liu et al. also reported that SM invasion was observed only in $61.8 \%$ of patients undergoing EFB and in $85 \%$ of patients undergoing TRUS-guided CNB (12).

There is much interest across the medical community regarding other potential diagnostic methods to predict SM invasion in rectal cancer. It is known that flat/ depressed lesions observed on conventional colonoscopy are associated with a higher risk of deep SM invasion $(15,16)$. The desmoplastic reaction associated with CRC is another useful histopathological predictor of SM invasion $(17,18)$. However, the accuracy of conventional colonoscopy in predicting SM invasion is limited to approximately $70-80 \%$ $(19,20)$. In contrast, the use of magnifying colonoscopy improves the discrimination rate of SM invasion to as high as $97 \%$ on the basis of identification of the Vn type pit pattern (21). Endoscopic ultrasonography (EUS) can predict SM invasion in early CRC (accuracy rate 93\%) with a high positive predictive value for deep SM invasion (22). However, conflicting data are available regarding the utility of EUS and magnifying colonoscopy for the management of CRC. Two studies that compared EUS with magnifying colonoscopy reported that EUS was superior to magnifying chromoendoscopy to determine the depth of tumor invasion [93\% vs. 59\% (22), and $93 \%$ vs. $63 \%$ (23)]. Interestingly, another study that investigated the diagnostic accuracy of magnifying colonoscopy and EUS in determining the depth of tumor invasion reported no differences between these modalities, particularly for non-polypoid lesions ( $85 \% v s$. $79 \%, \mathrm{P}=0.717$ ) (24).

Notably, the study reported by Liu et al. is a single-center retrospective study; therefore, a selection bias might have been introduced owing to the fact that patients enrolled might not have been consecutive cases. This would be a limitation of this research. Recently, endoscopic submucosal dissection (ESD) is used for accurate diagnosis and treatment of superficial colorectal lesions and subepithelial tumors. In this study, unnecessary surgery was performed on 8 adenomas, 8 HGINs, and 17 benign lesions. In the era of EUS, magnifying colonoscopy, and ESD, clinicians must weigh the risk-benefit ratio of TRUS-guided $\mathrm{CNB} v s$. the aforementioned modalities to predict SM cancer and the histopathological characteristics of subepithelial tumors. Nevertheless, the authors proved that TRUS-guided CNB could be a useful option for accurate preoperative diagnosis of rectal lesions. Prospective comparative studies are warranted in the future to compare TRUS-guided CNB with currently available modalities such as EUS, magnifying colonoscopy, and diagnostic ESD.

\section{Acknowledgments}

None.

\section{Footnote}

Conflicts of Interest: The authors have no conflicts of interest to declare.

Ethical Statement: The authors are accountable for all aspects of the work in ensuring that questions related to the accuracy or integrity of any part of the work are appropriately investigated and resolved.

\section{References}

1. Siegel RL, Miller KD, Jemal A. Cancer statistics, 2019. CA Cancer J Clin 2019;69:7-34.

2. Mohiuddin M, Regine WF, Marks G. Prognostic significance of tumor fixation of rectal carcinoma. Implications for adjunctive radiation therapy. Cancer 1996;78:717-22.

3. Kashida H, Kudo SE. Early colorectal cancer: concept, diagnosis, and management. Int J Clin Oncol 2006;11:1-8.

4. Kudo S, Kashida H, Tamura T, et al. Colonoscopic diagnosis and management of nonpolypoid early colorectal cancer. World J Surg 2000;24:1081-90.

5. Fenoglio CM, Kaye GI, Lane N. Distribution of human colonic lymphatics in normal, hyperplastic, and adenomatous tissue. Its relationship to metastasis from small carcinomas in pedunculated adenomas, with two case 
reports. Gastroenterology 1973;64:51-66.

6. Eckardt VF, Fuchs M, Kanzler G, et al. Follow-up of patients with colonic polyps containing severe atypia and invasive carcinoma. Compliance, recurrence, and survival. Cancer 1988;61:2552-7.

7. Tung SY, Wu CS. Clinical outcome of endoscopically removed early colorectal cancer. J Gastroenterol Hepatol 2003;18:1175-9.

8. Dixon MF. Gastrointestinal epithelial neoplasia: Vienna revisited. Gut 2002;51:130-1.

9. Park YJ, Kim WH, Paeng SS, et al. Histoclinical analysis of early colorectal cancer. World J Surg 2000;24:1029-35.

10. Shimomura T, Ishiguro S, Konishi H, et al. New indication for endoscopic treatment of colorectal carcinoma with submucosal invasion. J Gastroenterol Hepatol 2004;19:48-55.

11. Ishida H, Iwama T, Tomita N, et al. Diagnosis and management of hereditary colorectal cancer according to the JSCCR Guidelines 2012 for the Clinical Practice of Hereditary Colorectal Cancer. Nihon Rinsho 2014;72:143-9.

12. Liu M, Lu ZH, Wang QX, et al. Diagnostic value, safety, and histopathologic discrepancy risk factors for endoscopic forceps biopsy and transrectal ultrasound-guided core needle biopsy in rectum lesions. Ann Transl Med 2019;7:607.

13. Wei XB, Gao XH, Wang H, et al. More advanced or aggressive colorectal cancer is associated with a higher incidence of "high-grade intraepithelial neoplasia" on biopsy-based pathological examination. Tech Coloproctol 2012;16:277-83.

14. MacDonald AW, Tayyab M, Arsalani-Zadeh R, et al. Intramucosal carcinoma on biopsy reliably predicts invasive colorectal cancer. Ann Surg Oncol 2009; 16:3267-70.

15. Bond JH. Polyp guideline: diagnosis, treatment, and surveillance for patients with colorectal polyps. Practice

Cite this article as: Baek DH, Kim GH. Can transrectal ultrasound-guided core needle biopsy serve as an accurate diagnostic tool for rectal lesions? Ann Transl Med 2020;8(4):66. doi: 10.21037/atm.2019.11.144
Parameters Committee of the American College of Gastroenterology. Am J Gastroenterol 2000;95:3053-63.

16. Matsuda T, Parra-Blanco A, Saito Y, et al. Assessment of likelihood of submucosal invasion in non-polypoid colorectal neoplasms. Gastrointest Endosc Clin N Am 2010;20:487-96.

17. Ohno K, Fujimori T, Okamoto Y, et al. Diagnosis of desmoplastic reaction by immunohistochemical analysis, in biopsy specimens of early colorectal carcinomas, is efficacious in estimating the depth of invasion. Int $\mathrm{J}$ Mol Sci 2013;14:13129-36.

18. Desmouliere A, Guyot C, Gabbiani G. The stroma reaction myofibroblast: a key player in the control of tumor cell behavior. Int J Dev Biol 2004;48:509-17.

19. Tsuda SJS, INTESTINE. The usefulness of conventional endoscopy, barium enema, endoscopic ultrasonography and magnifying endoscopy for the diagnosis of depth of invasion in colorectal cancer. 2001;36:769-82.

20. Tsuruta O, Kawano H, Tsuji Y, et al. Effectiveness of magnifying endoscopy and endoscopic ultrasonography in diagnosing invasion depth of early colorectal cancer. Stomach Intestine 2001;36:791-9.

21. Oka S, Tanaka S, Kaneko I, et al. Diagnosis of the invasion depth using magnifying videocolonoscopy in early colorectal carcinoma. Early Colorectal Cancer 2005;9:161-8.

22. Hurlstone DP, Brown S, Cross SS, et al. High magnification chromoscopic colonoscopy or high frequency $20 \mathrm{MHz}$ mini probe endoscopic ultrasound staging for early colorectal neoplasia: a comparative prospective analysis. Gut 2005;54:1585-9.

23. Matsumoto T, Hizawa K, Esaki M, et al. Comparison of EUS and magnifying colonoscopy for assessment of small colorectal cancers. Gastrointest Endosc 2002;56:354-60.

24. Fu KI, Kato S, Sano Y, et al. Staging of early colorectal cancers: magnifying colonoscopy versus endoscopic ultrasonography for estimation of depth of invasion. Dig Dis Sci 2008;53:1886-92. 